Analyse de la littérature

\title{
Intérêts du réentraînement à l'effort dans la fibromyalgie et autres syndromes apparentés
}

\author{
D. Maquet ${ }^{\mathrm{a}, \mathrm{b}, *}$, C. Demoulin ${ }^{\mathrm{a}, \mathrm{b}}$, J.-L. Croisier ${ }^{\mathrm{a}, \mathrm{b}}$, J.-M. Crielaard ${ }^{\mathrm{a}, \mathrm{b}}$ \\ ${ }^{a}$ Département des sciences de la motricité, ISEPK, B21, université de Liège, 4, allée des Sports, 4000 Liège, Belgique \\ ${ }^{\mathrm{b}}$ CHU Sart-Tilman, Liège, Belgique
}

Reçu le 16 février 2007 ; accepté le 20 mars 2007

\begin{abstract}
Résumé
Objectif. - Rédiger un article de synthèse relatif au réentraînement à l'effort dans le cadre de la fibromyalgie et d'autres syndromes apparentés.

Matériel et méthodes. - La recherche bibliographique aborde (à partir de Medline via Ovid ${ }^{\circledR}$ et PubMed ${ }^{\circledR}$ ) les mots clés suivants : fibromyalgia; chronic fatigue syndrome; therapy; rehabilitation; aerobic; exercise; cognitive behavioral therapy. Une analyse de proche en proche est alors réalisée à partir des citations répertoriées.

Résultats. - De nombreux travaux ont évalué l'intérêt du réentraînement musculaire progressif dans le cadre de la prise en charge thérapeutique de la fibromyalgie ou d'autres syndromes apparentés. Bien que certaines revues systématiques n'aient pu établir sans équivoque l'efficacité de cette thérapeutique, la grande majorité des auteurs relatent les bénéfices apportés par ce réentraînement musculaire chez les patients souffrant de douleurs et/ou de fatigue chronique. Ce programme de réentraînement devra idéalement être proposé dans le cadre d'une approche thérapeutique multidisciplinaire. Le réentraînement musculaire tentera de lutter contre le déconditionnement classiquement mis en évidence chez les fibromyalgiques et visera dès lors à enrayer le cercle vicieux associant douleur, conduite d'évitement et d'inactivité, voire de kinésiophobie, déconditionnement, incapacité et détresse psychologique.

Conclusion. - Cette mise au point appuie l'intérêt d'un réentraînement musculaire progressif dans le traitement de la fibromyalgie et des syndromes associés. La mise en place de centres de revalidation, composés de praticiens capables d'apporter d'utiles réponses thérapeutiques à ces patients souffrant de douleurs et/ou de fatigue chronique, devrait aider à la prise en charge de ce problème de santé publique.
\end{abstract}

(C) 2007 Elsevier Masson SAS. Tous droits réservés.

Mots clés : Fibromyalgie ; Syndrome de fatigue chronique ; Revalidation ; Entraînement ; Approche thérapeutique

\section{Introduction}

La fibromyalgie (FM) se définit comme un syndrome polyalgique chronique et diffus, caractérisé par des douleurs musculosquelettiques (situées au-dessus et en dessous de la ceinture, mais aussi au niveau du squelette axial), présentes depuis au moins trois mois [93].

Ce syndrome s'accompagne le plus souvent d'un état généralisé d'allodynie, manifestation anormale de la nociception définie par la perception d'une douleur consécutive à un stimulus qui, normalement, ne devrait entrainer aucune réponse algogène [38].

\footnotetext{
${ }^{*}$ Auteur correspondant.

Adresse e-mail : d.maquet@ulg.ac.be (D. Maquet).
}

Les fibromyalgiques formulent fréquemment des plaintes additionnelles comportant la fatigue, les troubles du sommeil, la détresse psychologique, les douleurs articulaires, les spasmes musculaires et une raideur matinale [91]. La coexistence de la FM avec d'autres affections non musculosquelettiques, tels les troubles génito-urinaires, les céphalées de tension, la colite spasmodique ou encore le syndrome de « jambes sans repos » reste fréquente $[28,72,74,82,85,91,94]$.

La prévalence de la $\mathrm{FM}$, au sein de la population générale, apparaît extrêmement élevée et ce syndrome représente actuellement un véritable problème de santé publique, en raison d'un recours accru aux services des soins de santé et de la proportion élevée de fibromyalgiques bénéficiant d'une incapacité temporaire de travail, parfois supérieure à un an [41,42].

En 1981, Yunus et al. [96] précisaient déjà que $20 \%$ des patients consultant pour la première fois en rhumatologie pré- 
sentaient un syndrome fibromyalgique, devenant ainsi le diagnostic le plus souvent posé après la polyarthrite rhumatoïde [12,23]. En 1995, Wolfe et al. [92], après avoir observé un échantillon randomisé de 3000 personnes, estiment la prévalence de la FM à $2 \%$ de la population adulte nord-américaine et précisent une prévalence de $3,4 \%$ pour les sujets féminins et de $0,6 \%$ pour les sujets masculins. Une étude plus récente [87] signale une proportion de $3,3 \%$ de fibromyalgiques $(4,9 \%$ de femmes versus $1,6 \%$ d'hommes), majorée $(8,9 \%)$ chez les dames âgées de 50 à 64 ans. Le sex-ratio atteint le plus souvent $90 \%$ en faveur des femmes [92]. Le diagnostic de FM a déjà été posé chez des enfants [95]. Un niveau socioéconomique défavorable, une charge de travail physique élevée, un faible niveau d'instruction ou encore un statut de divorcé représentent quelques facteurs liés positivement à l'existence d'une FM [87]. L'aspect psychosocioculturel intervient certainement dans le vécu et l'« extériorisation»d'une douleur chronique [5].

Les praticiens sont de plus en plus amenés à traiter des patients qualifiés de chroniquement "douloureux et fatigués », un symptôme prédominant parfois sur l'autre. Le diagnostic de FM sera toujours posé après l'exclusion d'autres affections objectives (une pathologie rhumatismale, une maladie systémique, un trouble endocrinien, une anomalie du métabolisme calcique, une néoplasie sous-jacente) [90,94]. Les investigations devront, notamment, écarter diverses myalgies métaboliques, toxiques ou inflammatoires $[90,94]$.

La FM représente un syndrome, c'est-à-dire un ensemble de symptômes susceptibles de constituer une entité nosologique particulière. La FM se situe cependant aux frontières d'autres entités dont l'étiologie n'apparait toujours pas clairement: citons la spasmophilie [3] et le syndrome de fatigue chronique (SFC) $[24,36]$. À titre d'exemple, signalons qu'environ $70 \%$ des patients souffrant du SFC présentent des manifestations symptomatiques de fibromyalgie alors que $42 \%$ des patients fibromyalgiques répondent aux critères du SFC $[8,9,29-31,55$, 63] et, enfin, $37 \%$ des sujets SFC présentent les critères de la FM [15]. Wessely et al. [86] suggèrent que le SFC, la FM et le syndrome du côlon irritable constituent les manifestations polymorphes d'un même trouble somatique et psychologique.

\section{Matériel et méthodes}

Medline (via OVID ${ }^{\circledR}$ et PubMed ${ }^{\circledR}$ ) constitue la base des données bibliographiques nécessaires à la rédaction de cet article de synthèse. La recherche bibliographique aborde, de façon individuelle ou combinée, les mots clés suivants: fibromyalgia; chronic fatigue syndrome; therapy; rehabilitation; aerobic; exercise; cognitive behavioral therapy, et nous réalisons ensuite une analyse de proche en proche de la littérature.

\section{Approche thérapeutique et intérêts du réentraînement à l'effort}

L'étiopathogénie de la FM demeure obscure: plusieurs hypothèses physiopathologiques ont été suggérées, envisageant diverses anomalies musculaires, du sommeil, neuroendocriniennes, psychologiques, centrales et immunologiques, sans qu'aucune n'ait été formellement démontrée ; en effet, de telles modifications pourraient simplement correspondre à des manifestations secondaires au syndrome fibromyalgique.

En dépit d'une étiologie inconnue, une évaluation clinique rigoureuse ainsi qu'une définition d'objectifs thérapeutiques constituent les préalables indispensables à la détermination des meilleures options thérapeutiques [79]. Faut-il accepter la persistance d'une perception douloureuse ? Quels types d'activités convient-il de privilégier? Comment améliorer la qualité de vie et la capacité fonctionnelle des patients ? L'éducation du patient apparaît nécessaire afin d'éviter une surconsommation médicamenteuse [2].

Divers facteurs cognitifs, comme les croyances du patient liées aux schémas interprétatifs de leur situation douloureuse chronique, peuvent jouer un rôle essentiel dans l'expression de la symptomatologie. Ces croyances erronées - dramatisation, affection exclusivement irréversible, soulagement à partir des comportements d'évitement - constituent un obstacle pour participer à un programme de «réactivation». Smith et al. [73] démontrent que les distorsions cognitives en relation avec la douleur sont corrélées à l'incapacité ; dès lors, la prise en charge thérapeutique du fibromyalgique nécessitera une approche multidisciplinaire, proposant diverses interventions : information, suivi médical, stratégies cognitivocomportementales et reconditionnement physique, qui constituera un maillon essentiel de cette prise en charge thérapeutique.

En l'absence de traitement médicamenteux apportant aux fibromyalgiques des bénéfices évidents, la thérapeutique non médicamenteuse sera largement privilégiée [50]. Précisons cependant brièvement que certaines molécules ont fait l'objet d'études cliniques contrôlées et randomisées avec des résultats parfois contradictoires.

Les antidépresseurs tricycliques agissent, notamment au niveau sérotoninergique, voie située au carrefour du sommeil et de la perception de la douleur [56]. L'amitriptyline (10 à $25 \mathrm{mg}$ par jour) a démontré son efficacité sur la qualité du sommeil et le score de douleur de fibromyalgiques, alors que la fatigue répondrait mieux à la cyclobenzaprine (10 à $30 \mathrm{mg})$ présentant un effet myorelaxant plus marqué $[13,45]$. L'effet antalgique des antidépresseurs ne serait pas lié à la propre action antidépressive, aucune différence d'efficacité n'étant relevée entre patients déprimés ou non [60]. Les effets bénéfiques de ces molécules s'estompent au fil des mois, réduisant singulièrement leur intérêt, d'autant plus que ces médicaments entraînent des manifestations secondaires en relation, notamment avec leur action anticholinergique [17]. Les inhibiteurs sélectifs de la recapture de sérotonine (fluoxétine, citalopram) ne démontrent aucun effet analgésique; les troubles du sommeil, la dépression et l'humeur peuvent cependant être améliorés $[2,17]$. Un inhibiteur non sélectif de la recapture de la sérotonine (venlafaxine) entraîne chez $55 \%$ des fibromyalgiques présentant un désordre psychiatrique associé à (particulièrement la dépression et l'anxiété) une réduction des symptômes dont la fatigue $[2,22]$. 
Les benzodiazépines présentent un effet anxiolytique, une action myorelaxante et sédative. Cependant, ces molécules peuvent engendrer une dépendance physique et seront donc administrées avec précaution. L'alprazolam $(0,5-3 \mathrm{mg} / \mathrm{j}$, en traitement de courte durée) s'est révélé efficace chez des fibromyalgiques, lors d'une étude randomisée en double insu [50, 68,90].

La prise d'anti-inflammatoires non stéroïdiens ne semble guère efficace et jouerait un simple rôle coanalgésique dans le traitement de la FM $[2,68]$. L'ibuprofène s'avère plus efficace associé à l'alprazolam que prescrit seul, sans toutefois démontrer d'amélioration considérable [68]. L'association de naproxène et d'amitryptiline semble préférable au naproxène seul [68].

Crofford et al. [18] révèlent, dans une récente étude randomisée, en double insu, l'efficacité de la prégabaline $(450 \mathrm{mg} / \mathrm{j})$ dans l'amélioration de la douleur, de la fatigue et du sommeil en comparaison d'un groupe placebo.

L'administration de mélatonine chez le fibromyalgique n'améliore pas la qualité du sommeil en dépit de son rôle régulateur sur les différents cycles [62].

La kétamine, bloquant les récepteurs N-méthyl-D-aspartate (NMDA), a démontré son efficacité sur la perception douloureuse du fibromyalgique [35].

Les injections locales de lidocaïne limitées à trois ou quatre points sensibles $[7,49]$ entraînent une réduction des douleurs, plutôt attribuée aux effets mécaniques qu'au produit lui-même [37].

En complément d'une éventuelle prise en charge médicamenteuse à visée symptomatique, le reconditionnement physique semble clairement indiqué dans la prise en charge de la fibromyalgie. Diverses études, le plus souvent randomisées et contrôlées, ont apprécié l'intérêt de la revalidation progressive proposée isolément ou dans le cadre d'une approche multidisciplinaire.

En 1989, Bennett et al. [6] rapportent que $80 \%$ des fibromyalgiques présentent une condition physique anormalement faible. Cette observation motivera la réalisation d'études évaluant l'efficacité de divers programmes de revalidation. McCain et al. [51] démontrent les avantages d'un programme cardiovasculaire, en observant une augmentation du seuil de sensibilité douloureuse, une amélioration de la qualité de vie et de l'index cardiovasculaire ; en revanche, ils n'objectivent aucune modification de la qualité du sommeil et du score de l'échelle visuelle analogue (EVA) de la douleur. Mengshoel et al. [53] évaluent un programme bihebdomadaire de danse aérobique et rapportent après 20 semaines une amélioration de la seule endurance musculaire statique des membres supérieurs. Martin et al. [48] proposent un programme de six semaines, à concurrence de trois séances par semaine, d'activités aérobies, d'exercices de force et d'assouplissement, et observent une amélioration du score myalgique, de l'endurance et une réduction du nombre de points sensibles. Nichols et Glenn [58], lors d'un programme de marche de huit semaines proposé à un faible échantillon, rapportent exclusivement une amélioration des symptômes évalués à partir d'un questionnaire. Valim et al. [80] décrivent une amélioration de la consommation maximale d'oxygène, du seuil anaérobie, de la douleur, des aspects émotionnels et de la santé mentale après un entraînement aérobie de 20 semaines réalisé à raison de trois fois par semaine. Gowans et al. [34] rapportent une capacité physique améliorée de même que des troubles cognitifs et des symptômes somatiques réduits, un an après une revalidation aérobie de 23 semaines. Da Costa et al. [19] décrivent une amélioration fonctionnelle et une régression de la douleur chez des patients réalisant un programme d'exercices à domicile durant 12 semaines. Néanmoins, Dobkin et al. [21] rapportent des bénéfices plus conséquents lorsque l'entrainement est encadré par des professionnels de la santé, les exercices exécutés à domicile par les patients se réduisant au cours du temps. Tiidus et al. [76] ainsi que Dawson et al. [20] proposent un programme d'entraînement (aérobie, force et étirements), et mesurent une amélioration significative de l'humeur, du nombre de points sensibles et de la vitesse de marche, sans néanmoins objectiver une amélioration de la capacité de travail et de la flexibilité. Rooks et al. [67] observent, après 20 semaines d'entraînement (exercices de force et cardiorespiratoires), une amélioration de la force, du test de marche sur six minutes et des scores au Fibromyalgia Impact Questionnaire (FIQ). En outre, ces auteurs rapportent une très bonne adhésion et tolérance des fibromyalgiques sélectionnés à ce programme. McCain et al. [51] comparent un programme d'entraînement aérobie durant 20 semaines à un programme d'étirements, et observent une amélioration des qualités physiques et du seuil de sensibilité à la douleur. Les auteurs n'objectivent aucun bénéfice sur l'intensité de la douleur et les troubles du sommeil. Wigers et al. [88] comparent un programme d'exercices aérobies à un programme de gestion du stress et à une prise en charge classique en secteur de santé primaire. Ce travail met en évidence des bénéfices supérieurs en termes de fonctionnalité, de capacité de travail et de douleur (intensité et localisation) en faveur des fibromyalgiques participant au programme d'entraînement aérobie. Martin et al. [48] comparent un programme « aérobie-étirements » à un programme de relaxation et démontrent des bénéfices supérieurs associés au programme d'exercices. Verstappen et al. [84] relatent également une progression de la fonction physique de fibromyalgiques au terme d'une période d'entraînement.

D'autres études évaluent l'intérêt d'un réentraînement à l'effort du fibromyalgique dans le cadre d'une approche multidisciplinaire. Nielson et al. [59] proposent une prise en charge de trois semaines (relaxation assistée par biofeedback, exercices aérobies, étirements, thérapie cognitivocomportementale, éducation, gestion de la douleur) et observent une régression de la douleur, une amélioration du contrôle de la douleur, des composantes émotionnelles et de la fonction. Les auteurs observent des bénéfices maintenus après 30 mois [88]. Turk et al. [79] mesurent une amélioration (maintenue à six mois) en termes de sévérité de la douleur, de l'anxiété, de la dépression, de la détresse et de la fatigue dans le décours d'une approche multidisciplinaire (éducation, exercices fonctionnels et thérapie cognitivocomportementale). Pfeiffer et al. [61] relatent une amélioration significative des scores au questionnaire 
FIQ après un programme intensif (réalisé durant trois demijournées) comportant de la thérapie cognitivocomportementale, des exercices et des informations éducationnelles. Bailey et al. [1] mesurent, au terme d'une approche multidisciplinaire de 12 semaines (éducation et exercices), une amélioration de la fonction physique générale.

Le réentraînement musculaire a également été proposé dans la prise en charge des syndromes apparentés à la FM et semble dès lors représenter un élément essentiel de la prise en charge thérapeutique du patient souffrant du SFC.

Fulcher et White [25] comparent, chez 66 sujets souffrant du SFC, un programme aérobie quotidien (vélo, marche, natation) à raison de 30 minutes par jour à une intensité de $60 \%$ de la consommation maximale d'oxygène, versus un programme de relaxation et d'étirements (30 minutes par jour, cinq fois par semaine); ils démontrent une amélioration conséquente du bien-être des sujets du groupe «exercice», comparativement à ceux du groupe «étirements et relaxation» $(55 \%$ des patients du groupe « exercice» contre $27 \%$ de ceux du groupe " étirements-relaxation ») et précisent un effet favorable des exercices sur la fatigue et la fonction physique. Powell et al. [64] comparent l'influence d'un programme éducationnel (interventions visant à encourager la pratique d'exercices physiques) et d'une information écrite (groupe témoin). Après un an, l'intervention éducationnelle améliore significativement la fonction physique, la fatigue, l'humeur et le sommeil des patients comparativement au groupe témoin.

Par ailleurs, aucune étude n'apprécie l'influence d'un repos prolongé sur la symptomatologie des patients souffrant du SFC. Cependant, l'observation symptomatique de sujets sains ou de sujets ayant présenté une infection virale suggère que le repos perpétuerait et même accentuerait la fatigue [66].

\section{Discussion}

La proposition thérapeutique d'un réentraînement musculaire progressif peut sembler paradoxale chez des patients en permanence «douloureux» et/ou «fatigués ». Néanmoins, le déconditionnement musculaire est classiquement mis en évidence chez les fibromyalgiques $[6,46]$, et dès lors cet entraînement visera à enrayer le cercle vicieux associant la douleur, les conduites d'évitement et d'inactivité, voire de kinésiophobie, le déconditionnement, l'incapacité et la détresse psychologique. En effet, la douleur, dont l'expression et la modulation sont régulièrement influencées par divers aspects psychologiques, comportementaux [78] et socioéconomiques [5], entrainerait un comportement d'évitement, voire d'inactivité totale. Cette situation provoque un véritable déconditionnement s'accompagnant de modifications bioénergétiques pérennisant l'inactivité et explique la réaction douloureuse exacerbée lors d'une reprise (hypothétique) d'activités. L'incapacité professionnelle, de loisir et même domestique résultante, apparaît dès lors d'autant plus difficile à gérer qu'elle demeure inexpliquée par des éléments somatiques probants, favorisant dans le chef du patient le développement progressif d'un sentiment de culpabilité pouvant éventuellement le mener à la dépression.
De multiples études menées depuis plus d'une décennie confirment l'intérêt d'un réentrainement, même si certaines revues systématiques de la littérature $[11,71,72]$ n'ont pu établir sans équivoque l'efficacité de cette thérapeutique.

Cette revalidation pourra être précédée d'une évaluation algofonctionnelle (incluant par exemple diverses épreuves physiques, une quantification de la douleur et divers questionnaires de qualité de vie, d'anxiété et de dépression), afin d'envisager un suivi longitudinal rigoureux [47].

Le type et la progression des activités proposées, la durée, la fréquence et l'intensité des séances représentent des éléments essentiels de la revalidation du fibromyalgique $[43,69]$.

Une fréquence hebdomadaire d'entraînement de trois séances, conformément aux recommandations du Collège américain de médecine du sport (1999), semble adéquate. Plusieurs auteurs signalent la difficulté pour un fibromyalgique de participer à plus de trois séances hebdomadaires $[53,54,89]$. L'intensité de l'effort proposé correspond le plus souvent à un entraînement aérobie et pourra dès lors se situer entre 60 et $75 \%$ de la FC maximale théorique, ce qui autorise une progression régulière tout en évitant le risque de surentrainement [16]. Le patient devra néanmoins être informé d'une possible aggravation transitoire de la symptomatologie douloureuse durant les premières séances. Mc Cain et al. [51] préconisent des efforts plus intenses (FC d'entraînement supérieure à $150 \mathrm{bpm}$ ), réputés favorables à la libération de bêtaendorphines $[32,44,70]$, de somathormone $[27,39,83]$ ou encore de sérotonine $[14,26,40,51]$; l'absence d'amélioration de la douleur peut s'expliquer selon eux par une intensité d'entrainement trop faible. D'autres auteurs ont cependant rapporté une exacerbation de la douleur et une dégradation du score FIQ lors d'un programme trop intense $[54,81]$. Les exercices proposés peuvent correspondre à des activités aérobies diverses, comportant la marche sur tapis roulant, la bicyclette ergométrique, le rameur et le step, ainsi que des exercices dynamiques des membres supérieurs et inférieurs. La relaxation et les étirements permettent de lutter respectivement contre un tonus musculaire excessif et un manque de flexibilité. La participation régulière aux séances d'entraînement des fibromyalgiques devra être encouragée.

Par ailleurs, le taux d'abandon de sujets indemnes de toute pathologie atteint parfois $50 \%$ à six mois [43]. Une étude de Wigers et al. [89] sur le reconditionnement du fibromyalgique a d'ailleurs dû se terminer prématurément par manque de participants. Dès lors, malgré la proximité géographique d'un centre de reconditionnement et la motivation des patients, une participation régulière demeure parfois difficile, particulièrement lorsque les patients sont invités à poursuivre de manière autonome leur revalidation [89].

L'amélioration des performances musculaires s'expliquerait par diverses adaptations classiquement observées dans les suites d'un entraînement : citons l'hypertrophie des fibres lentes, l'accroissement de la densité capillaire et mitochondriale, l'élévation de la concentration de myoglobine facilitant la diffusion de l'oxygène vers le compartiment cytoplasmique $[43$, 
58]. L'exercice physique modifie favorablement d'autres paramètres :

- la régression de l'anxiété et de l'état dépressif [16,51];

- l'augmentation du seuil de sensibilité douloureuse [44];

- la tolérance accrue des symptômes $[16,44]$;

- la libération d'endorphines par stimulation du système opioïde endogène [44] survenant au-delà du seuil aérobie ou lors d'efforts dépassant 60 minutes [70], mais qui pourrait se manifester lors d'exercices moins intenses pratiqués par des personnes particulièrement déconditionnées [32];

- l'amélioration de l'humeur, du bien-être, de la confiance en ses possibilités individuelles mais aussi la diminution du sentiment d'impuissance $[33,57,65,75]$;

- l'amélioration de la qualité du sommeil $[51,77]$.

La revalidation collective offre aux fibromyalgiques la possibilité de discuter entre eux et surtout d'être mieux compris par des professionnels de la santé. La thérapie comportementale, destinée à modifier de façon durable les comportements défavorables, à apprendre à gérer les symptômes, à faire face à l'affection et à maintenir les activités physiques, constitue une approche complémentaire intéressante $[4,10,52,69,79]$.

Le programme de reconditionnement physique devrait se poursuivre au moins 12 semaines afin d'apporter aux fibromyalgiques les bénéfices escomptés [10,48]. Le maintien à long terme de ces bénéfices dépendra de la poursuite d'activités physiques. Les résultats apparaissent décevants chez les patientes interrompant leurs activités physiques [33,89], alors que les données restent très encourageantes pour les fibromyalgiques poursuivant de manière autonome des efforts de durée, de fréquence et d'intensité suffisantes [89].

En dépit de mécanismes physiopathologiques controversés, soulignons que poser le diagnostic permet d'éliminer la notion de "malade imaginaire » et d'accepter la réalité du mal-être, démarche favorable à l'établissement d'une relation de confiance entre le praticien et son patient. La prise en charge, précédée d'une évaluation des facteurs somatiques, psychosociaux et comportementaux, se réalisera idéalement au sein d'une équipe pluridisciplinaire de praticiens partageant des concepts thérapeutiques communs et cohérents. Le traitement sera souvent compliqué par des sentiments «d'inefficacité personnelle», de découragement, voire d'agressivité, induits par les échecs multiples, les frustrations, ... Les options thérapeutiques guideront le patient vers une stratégie active. Le reconditionnement physique représentera une composante essentielle, il privilégiera l'amélioration fonctionnelle en vue d'une éventuelle diminution secondaire de la douleur. La reconceptualisation des croyances erronées du fibromyalgique et l'acquisition de compétences (relaxation, stratégies d'adaptation à la stimulation nociceptive, planification réaliste de tâches, gestion du stress,...) contribueront à la suppression des attitudes défavorables au profit de comportements plus adaptés indispensables à la pérennité et à la progression des bénéfices $[5,49,69]$.

\section{Conclusion}

Cette mise au point met en évidence l'intérêt d'un réentraînement musculaire progressif dans le cadre du traitement de la fibromyalgie.

La mise en place de centres de revalidation, composés de praticiens capables d'apporter d'utiles réponses à ces patientes souffrant de douleurs chroniques, devrait aider à la prise en charge de ce problème de santé publique.

\section{Références}

[1] Bailey A, Starr L, Alderson M, Moreland J. A comparative evaluation of a fibromyalgia rehabilitation program. Arthritis Care Res 1999;12:33640.

[2] Barkhuizen A. Rational and targeted pharmacologic treatment of fibromyalgia. Rheum Dis Clin North Am 2002;28:261-90.

[3] Bastin JM. La spasmophilie. Acta Rheumatol Belg 1981;5:41-8.

[4] Bennett RM. Fibromyalgia and the disability dilemma. A new era in understanding a complex, multidimensional pain syndrome. Arthritis Rheum 1996;39:1627-34.

[5] Bennett RM. The rational management of fibromyalgia patients. Rheum Dis Clin North Am 2002;28:181-99.

[6] Bennett RM, Clark SR, Goldberg L, Nelson D, Bonafede RP, Porter J, et al. Aerobic fitness in patients with fibrositis. A controlled study of respiratory gas exchange and 133-xenon clearance from exercising muscle. Arthiritis Rheum 1989;32:454-60.

[7] Borg-Stein J, Stein J. Trigger points and tender points: one and the same? Does injection treatment help? Rheum Dis Clin North Am 1996;22:30522.

[8] Buchwald D, Garrity D. Comparison of patients with chronic fatigue syndrome, fibromyalgia and multiple chemical sensitivities. Arch Intern Med 1994;154:2049-53.

[9] Buchwald D. Fibromyalgia and chronic fatigue syndrome: similarities and differences. Rheum Dis Clin North Am 1996;22:219-43.

[10] Burckhardt CS, Mannerkorpi K, Hedenberg L, Bjelle A. A randomized, controlled clinical trial of education and physical training for women with fibromyalgia. J Rheumatol 1994;21:714-20.

[11] Busch AJ, Schachter CL, Peloso PM. Fibromyalgia and exercise training: a systematic review of randomized controlled trials. Phys Ther Rev 2001; 6:287-306.

[12] Buskila D. Fibromyalgia, chronic fatigue syndrome, and myofascial pain syndrome. Curr Opin Rheumatol 2001;13:117-27.

[13] Carette S, Bell MJ, Reynolds WJ, Haraoui B, Mc Cain GA, Bykerk VP, et al. Comparison of amitriptyline, cyclobenzaprine, and placebo in the treatment of fibromyalgia. A randomized, double blind clinical trial. Arthritis Rheum 1994;37:32-40.

[14] Chaouloff F. Physical exercises and brain monoamines: a review. Acta Physiol Scan 1989;137:1-13.

[15] Ciccone DS, Natelson BH. Comorbid illness in women with chronic fatigue syndrome: a test of the single syndrome hypothesis. Psychosom Med 2003;65:268-75.

[16] Clark SR, Jones KD, Burckhardt CS, Bennett RM. Exercise for patients with fibromyalgia: risks versus benefits. Curr Rheumatol Rep 2001;3: $135-46$.

[17] Cortet B, Houvenagel E, Forzy G, Vincent G, Delcambre B. Évaluation de l'efficacité d'un traitement sérotoninergique (Chlorhydrate de fluoxétine). Étude ouverte au cours de la fibromyalgie. Rev Rhum Mal Osteoartic 1992;59:497-500.

[18] Crofford LJ, Rowbotham MC, Mease PJ, Russell IJ, Dworkin RH, Corbin $\mathrm{AE}$, et al. Pregabalin for the treatment of fibromyalgia syndrome: results of a randomized, double blind, placebo-controlled trial. Arthritis Rheum 2005;52:1264-73.

[19] Da Costa D, Abrahamowicz M, Lowensteyn I, Bernatsky S, Dritsa M, Fitscharles MA, et al. A randomised clinical trial of an individualized 
home-based exercise programme for women with fibromyalgia. Rheumatol (Oxford) 2005;44:1422-7.

[20] Dawson KA, Tiidus PM, Pierrynowski M, Crawford JP, Trotter J. Evaluation of a community-based exercise program for diminishing symptoms of fibromyalgia. Physiother Can 2003;55:17-22.

[21] Dobkin PL, Abrahamowicz M, Fitzcharles MA, Dritsa M, da Costa D. Maintenance of exercise in women with fibromyalgia. Arthritis Rheum 2005;53:724-31.

[22] Dwight MM, Arnold LM, O'brien H, Metzger R, Morris-Park E, Keck Jr. PE. An open clinical trial of venlafaxine treatment of fibromyalgia. Psychosomatics 1998;39:14-7.

[23] Fitzcharles MA. Is fibromyalgia a distinct clinical entity? The approving rheumatologist's evidence. Baillieres Best Pract Res Clin Rheumatol 1999;13:437-43.

[24] Fukuda K, Straus SE, Hickie I, Sharpe MC, Dobbins JG, Komaroff A. The chronic fatigue syndrome: a comprehensive approach to its definition and study. International Chronic Fatigue Syndrome Study Group. Ann Intern Med 1994;121:953-9.

[25] Fulcher KY, White PD. A randomized controlled trial of graded exercise therapy in patients with the chronic fatigue syndrome. BMJ 1997;314: $1647-52$.

[26] Geel SE. The fibromyalgia syndrome: musculoskeletal pathophysiology. Semin Arthritis Rheum 1994;23:347-53.

[27] Geenen R, Jacobs JWG, Bijlsma JWJ. Evaluation and management of endocrine dysfunction in fibromyalgia. Rheum Dis Clin N Am 2002;28: 389-404.

[28] Gerster JC. Fibromyalgie. Le passé, le présent. Rev Med Suisse Rom 1999;199:513-6.

[29] Goldenberg DL, Simms RW, Geiger A, Komaroff AL. High frequency of fibromyalgia in patients with chronic fatigue seen in a primary care practice. Arthritis Rheum 1990;33:381-7.

[30] Goldenberg DL. Fibromyalgia and its relation to chronic fatigue syndrome, viral illness and immune abnormalities. J Rheumatol 1989;16 (Suppl 19):S91-3.

[31] Goldenberg DL. Fibromyalgia, chronic fatigue syndrome, and myofascial pain. Curr Opin Rheumatol 1996;8:113-23.

[32] Goldfarb AH, Jamurtas AZ. Beta-endorphin response to exercise. An update. Sports Med 1997;24:8-16.

[33] Gowans SE, De Hueck A, Voss S, Richardson M. A randomized, controlled trial of exercise and education for individuals with fibromyalgia. Arthritis Care Res 1999;12:120-8.

[34] Gowans SE, Dehueck A, Voss S, Silaj A, Abbey SE. Six-month and oneyear follow-up of 23 weeks of aerobic exercise for individuals with fibromyalgia. Arthritis Rheum 2004;51:890-8.

[35] Graven-Nielsen T, Aspegren KS, Henriksson KG, Bengtsson M, Sorensen J, Johnson A, et al. Ketamine reduces muscle pain, temporal summation, and referred pain in fibromyalgia patients. Pain 2000;85:483-91.

[36] Holmes GP, Kaplan JE, Gantz NM, Komaroff AL, Schonberger LB, Straus SE, et al. Chronic fatigue syndrome: a working case definition. Ann Intern Med 1988;108:387-9.

[37] Hong CZ, Hsueh TC. Difference in pain relief after trigger point injections in myofascial pain patients with and without fibromyalgia. Arch Phys Med Rehabil 1996;77:1161-6.

[38] Houvenagel E. Les mécanismes de la douleur de la fibromyalgie. Rev Rhum 1999;66:105-10.

[39] Jenkins PJ. Growth hormone and exercise: physiology, use and abuse. Growth Horm IGF Res 2001;11:71-7.

[40] Jouvet M. Biogenic amines and the states of sleep. Science 1969;163:3241.

[41] Kissel JT. The problem of fibromyalgia. Muscle Nerv 2002;25:473-6.

[42] Kissel W, Mahnig P. Die fibromyalgie in der begutachtungssituation. Praxis 1998;87:538-45.

[43] Klug GA, McAuley E, Clark S. Factors influencing the development and maintenance of aerobic fitness: Lessons applicable to the fibrositis syndrome. J Rheumatol 1989;16:30-9.

[44] Koltyn KF. Analgesia following exercise: a review. Sports Med 2000;29: $85-9$.

[45] Lautenschlager J. Present state of medication therapy in fibromyalgia syndrome. Scand J Rheumatol 2000;113:32-6.
[46] Maquet D, Croisier JL, Renard C, Crielaard JM. Muscle performance in patients with fibromyalgia. Joint Bone Spine 2002;69:293-9.

[47] Maquet D, Croisier JL, Demoulin C, Faymonville M, Crielaard JM. Intérêt de la revalidation aérobie dans la prise en charge de la fibromyalgie. Rev Med Liege 2006;61:109-16.

[48] Martin L, Nutting A, MacIntosh BR, Edworthy SM, Butterwick D, Cook J. An exercise program in the treatment of fibromyalgia. J Rheumatol 1996;23:1050-3.

[49] Masi AT, Yunus MB. Fibromyalgia: which is the best treatment? A personalized, comprehensive, ambulatory, patient-involved management programme. Baillieres Clin Rheumatol 1991;4:333-70.

[50] McCain GA. A cost-effective approach to the diagnosis and treatment of fibromyalgia. Rheum Dis Clin North Am 1996;22:323-49.

[51] McCain GA, Bell DA, Mai FM, Halliday PD. A controlled study of the effects of a supervised cardiovascular fitness-training program on the manifestations of primary fibromyalgia. Arthritis Rheum 1988;31:113541.

[52] Mengshoel AM, Forseth KO, Haugen M, Walle-Hansen R, Forre O. Multidisciplinary approach to fibromyalgia. A pilot study. Clin Rheumatol 1995; $14: 165-70$.

[53] Mengshoel AM, Komnoes HB, Forre O. The effects of physical fitness training in female patients with fibromyalgia. Clin Exp Rheumatol 1992; 10:345-9.

[54] Meyer BB, Lemley KJ. Utilizing exercise to affect the symptomology of fibromyalgia: a pilot study. Med Sci Sports Exerc 2000;32:1691-7.

[55] Moldofsky H. Fibromyalgia, sleep disorder and chronic fatigue syndrome. Ciba Found Symp 1993;173:262-71.

[56] Moldofsky H. Management of sleep disorders in fibromyalgia. Rheum Dis Clin North Am 2002;28:353-65.

[57] Moses J, Steptoe A, Mathews A, Edwards S. The effects of exercise training on mental well-being in the normal population: a controlled trial. J Psychosom Res 1989;33:47-61.

[58] Nichols DS, Glenn TM. Effects of aerobic exercise on pain perception, affect, and level of disability in individuals with fibromyalgia. Phys Ther 1994;74:327-32.

[59] Nielson WR, Walker C, McCain GA. Cognitive behavioural treatment of fibromyalgia syndrome: Preliminary findings. J Rheumatol 1992;19:98103.

[60] Onghena P. Antidepressant-induced analgesia in chronic non-malignant pain: a meta-analysis of 39 placebo-controlled studies. Pain 1992;49: 209-19.

[61] Pfeiffer A, Thompson JM, Nelson A, Tucker S, Luedtke C, Finnie S, et al. Effects of a 1.5 days multidisciplinary outpatient treatment program for fibromyalgia. A pilot study. Am J Phys Med Rehabil 2003;82:18691.

[62] Pillemer SR, Bradley LA, Crofford LJ, Moldofsky H, Chrousos GP. The neuroscience and endocrinology of fibromyalgia. Arthritis Rheum 1997; 40:1928-39.

[63] Poller C, Natelson BH, Lange G, Tiersky L, Deluca J, Policastro T, et al. Medical evaluation of Persian Gulf veterans with fatigue and/or chemical sensitivity. J Med 1998;29:101-13.

[64] Powell P, Bentall RP, Nye FJ, Edwards RH. Randomised controlled trial of patient education to encourage graded exercise in chronic fatigue syndrome. BMJ 2001;322:387-90.

[65] Ramsay C, Moreland J, Ho M, Joyce S, Walker S, Pullar T. An observerblinded comparison of supervised and unsupervised aerobic exercise regimens in fibromyalgia. Rheumatology (Oxford) 2000;39:501-5.

[66] Reid S, Chalder T, Cleare A, Hotopf M, Wessely S. Chronic fatigue syndrome. Clin Evid 2002;8:1075-88.

[67] Rooks DS, Silverman CB, Kantrowitz FG. The effects of progressive strength training and aerobic exercise on muscle strength and cardiovascular fitness in women with fibromyalgia: a pilot study. Arthritis Rheum 2002;47:22-8.

[68] Russell IJ, Fletcher EM, Michalek JE, Mc Broom PC, Hester GG. Treatment of primary fibrositis/fibromyalgia syndrome with ibuprofen and alprazolam: a double blind, placebo-controlled study. Arthritis Rheum 1991;34:552-60. 
[69] Sandstrom MJ, Keefe FJ. Self-management of fibromyalgia: the role of formal coping skills training and physical exercise training programs. Arthritis Care Res 1998;11:432-47.

[70] Schwarz L, Kindermann W. Changes in beta-endorphin levels in response to aerobic and anaerobic exercise. Sports Med 1992;13:25-36.

[71] Sim J, Adams N. Systematic review of randomised controlled trials of nonpharmacological interventions for fibromyalgia. Clin J Pain 2002;18: 324-36.

[72] Sivri A, Cindas A, Dincer F, Sivri B. Bowel dysfunction and irritable bowel syndrome in fibromyalgia patients. Clin Rheumatol 1996;15:2836.

[73] Smith TH, Follick MJ, Abers DK, Adams A. Cognitive distortion and disability in chronic low back pain. Cognitive Res 1986;10:201-10.

[74] Sperber AD, Atzmon Y, Neumann L, Weisberg I, Shalit Y, Abu-Shakrah $\mathrm{M}$, et al. Fibromyalgia in the irritable bowel syndrome: studies of prevalence and clinical implications. Am J Gastroenterol 1999;94:3541-6.

[75] Taylor CB, Sallis JF, Needle R. The relation of physical activity and exercise to mental health. Publ Health Rep 1985;100:195-202.

[76] Tiidus PM, Pierrynowski M, Dawson KA. Influence of moderate training on gait and work capacity of fibromyalgia patients: a preliminary field study. J Sports Sci Med 2002;1:122-7.

[77] Torsvall L, Akerstedt T, Lindbeck G. Effects on sleep stages and EEG power density of different degrees of exercise in fit subjects. Electroencephalogr Clin Neurophysiol 1984;57:347-53.

[78] Turk DC, Monarch ES, Williams AD. Psychological evaluation of patients diagnosed with fibromyalgia syndrome: a comprehensive approach. Rheum Dis Clin North Am 2002;28:219-33.

[79] Turk DC, Okifuji A, Sinclair JD, Starz TW. Interdisciplinary treatment for fibromyalgia syndrome: clinical and statistical significance. Arthritis Care Res 1998;11:186-95.

[80] Valim V, Oliveira L, Suda A, Silva L, de Assis M, Barros Neto T, et al. Aerobic fitness effects in fibromyalgia. J Rheumatol 2003;30:1060-9.

[81] Van Santen M, Bolwijn P, Verstappen F, Bakker C, Hidding A, Houben $\mathrm{H}$, et al. High or low aerobic fitness training in fibromyalgia: does it matter? J Rheumatol 2002;29:575-81.

[82] Veale D, Kavanagh G, Fielding JF, Fitzgerald O. Primary fibromyalgia and the irritable bowel syndrome: different expressions of a common pathogenetic process. Br J Rheumatol 1991;30:220-2.
[83] Veldhuis JD, Iranmanesh A, Weltman A. Elements in the pathophysiology of diminished growth hormone $(\mathrm{GH})$ secretion in aging humans. Endocrine 1997;7:41-8.

[84] Verstappen FTJ, van Santen-Hoeuftt HMS, Bolwijn PH, van der Linden $\mathrm{S}$, Kuipers $\mathrm{H}$. Effects of a group activity program for fibromyalgia patients on physical fitness and well being. J Musculoskel Pain 1997;5: $17-28$.

[85] Waxman J, Zatzakis SM. Fibromyalgia and menopause: examination of the relationship. Postgrad Med 1986;80:165-71.

[86] Wessely S, Nimnuan C, Sharpe M. Functional somatic syndromes: one or many? Lancet 1999;354:936-9.

[87] White KP, Speechley M, Harth M, Ostbye T. The London fibromyalgia epidemiology study: The prevalence of fibromyalgia syndrome in London, Ontario. J Rheumatol 1999;26:1570-6.

[88] White KP, Nielson WR. Cognitive behavioural treatment of fibromyalgia syndrome: a follow-up assessment. J Rheumatol 1995;22:717-21.

[89] Wigers GH, Stiles TC, Vogel PA. Effects of aerobic exercise versus stress management treatment in fibromyalgia: a 4.5 -year prospective study. Scand J Rheumatol 1996;25:77-86.

[90] Wilke WS. Fibromyalgia. Recognizing and addressing the multiple interrelate factors. Postgrad Med 1996;100:153-70.

[91] Winfield JB. Pain in fibromyalgia. Rheum Dis Clin North Am 1999;25: $55-79$.

[92] Wolfe F, Ross K, Anderson J, Russell IJ, Hebert L. The prevalence and characteristics of fibromyalgia in the general population. Arthritis Rheum 1995;38:19-28.

[93] Wolfe F, Smythe HA, Yunus MB, Bennett RM, Bombardier C, Goldenberg DL, et al. The American College of Rheumatology 1990 criteria for the classification of fibromyalgia. Arthritis Rheum 1990;33:160-73.

[94] Yunus MB. A comprehensive medical evaluation of patients with fibromyalgia syndrome. Rheum Dis Clin North Am 2002;28:201-17.

[95] Yunus MB, Masi A. Juvenile primary fibromyalgia syndrome. A clinical study of thirty-three patients and matched normal controls. J Rheumatol 1985;12:636-7.

[96] Yunus MB, Masi AT, Calabro JJ, Miller KA, Feigenbaum SL. Primary fibromyalgia (fibrositis): clinical study of 50 patients with matched normal controls. Semin Arthritis Rheum 1981;11:151-71. 